\title{
Diagnosis and management of spinal tuberculosis combined with brucellosis: A case report and literature review
}

\author{
DEXIN ZOU ${ }^{1,2}$, JUNLIN ZHOU ${ }^{1}$ and XIAOBING JIANG ${ }^{3}$ \\ ${ }^{1}$ Department of Orthopaedics, Beijing Chaoyang Hospital, Capital Medical University, \\ Beijing 100020; ${ }^{2}$ Department of Orthopaedics, Yantai Yantaishan Hospital; ${ }^{3}$ Department of Equipment, \\ Yantai Yuhuangdi Hospital, Yantai, Shandong 264000, P.R. China
}

Received December 20, 2015; Accepted February 1, 2017

DOI: $10.3892 / \mathrm{etm} .2018 .5812$

\begin{abstract}
Tuberculosis combined with brucellosis is a very rare condition. Overlapping clinical presentation and laboratory parameters of tuberculosis and brucellosis may lead to misdiagnosis or delayed diagnosis of the condition. The current study presents the case of a 45-year-old male with symptoms of lower back pain, non-tender swelling in the right flank, intermittent hyperpyrexia, sweating, body aches and numbness and weakness of right lower limb. A lumbar computed tomograph (CT) scan and magnetic resonance imaging indicated vertebral destruction and the formation of sequestra and thecal sac compression. Tuberculosis was suspected, but subsequent culture of blood and pus revealed the co-infection of Mycobacterium tuberculosis and Brucella melitensis. The patient was treated with antibiotics, CT-guided percutaneous drainage of the abscess and posterior approach decompression, debridement, instrumentation and fusion. Co-existence of spinal tuberculosis and brucellosis is rare and clinicians should strengthen the awareness of such conditions in similar patients. CT-guided percutaneous drainage is effective in the diagnosis and management of spinal tuberculosis with abscess.
\end{abstract}

\section{Introduction}

Tuberculosis (TB) is caused by infection of Mycobacterium tuberculosis (M.tuberculosis), which remains the leading cause of mortality from infectious disease worldwide (1). According to a World Health Organization

Correspondence to: Mrs. Junlin Zhou, Department of Orthopaedics, Beijing Chaoyang Hospital, Capital Medical University, 8 Worker's Stadium South Road, Beijing 100020, P.R. China

E-mail: zhoujunlin119@163.com

Mrs. Xiaobing Jiang, Department of Equipment, Yantai Yuhuangdi Hospital, 20 Yu Dong Road, Yantai, Shandong 264000, P.R. China E-mail: 61647741@qq.com

Key words: spinal tuberculosis, brucellosis, psoas abscess, percutaneous drainage, posterior debridement study in 2012, TB remains a global emergency, particularly in less developed countries (2). Tuberculosis typically affects the pulmonary system but may also affect any other organ system within the body; this type of TB is referred to as extra-pulmonary TB (3). Extra-pulmonary involvement accounts for approximately $14 \%$ of TB patients and skeletal system involvement occurs in $1-3 \%$ of patients $(4,5)$. Spinal tuberculosis is the most frequent bone localization, accounting for $40-50 \%$ of all skeletal tuberculosis cases, followed by major weight bearing joints of the lower extremity, including the hip and knee (6-8). Extra-pulmonary TB often occurs in patients with reduced immune function. It is well known that the human immunodeficiency virus (HIV) is the etiological agent responsible for acquired immune deficiency syndrome. It severely weakens the immune system and increases the susceptibility of individuals to opportunistic infections, including TB (9). TB may cause macrophage dysfunction, anemia and hypoproteinemia, thus making patients highly vulnerable to other infections. Brucellosis (BS) is a zoonotic bacterial disease, caused by the gram-negative bacteria Brucella (10). The involvement of the musculoskeletal is associated with $10-85 \%$ of complications associated with brucellosis (11). Similarly to TB, spinal involvement is one of the most commonly encountered forms of human brucellosis (12). BS may be misdiagnosed as TB as its clinical features and basic laboratory parameters are comparable. It is especially difficult to make definitive diagnoses in cases of co-infection with TB and BS. The present study reports a case of spinal TB in association with BS. To the best of our knowledge, this co-infection has not been described in previous literature.

\section{Case report}

History and physical examination. A 45-year-old male was admitted as an outpatient to the Department of Orthopedics, Yantaishan Hospital (Yantai, China) with a history of lower back pain and non-tender swelling in the right flank for 3 months, intermittent hyperpyrexia, sweating and body aches for 3 weeks and numbness and weakness of right lower limb for 2 days. The patient had been in excellent health prior to this presentation with no other remarkable medical or surgical history. There was no medical history of personal or family tuberculosis. The patient also had no history of tobacco use, 
intravenous drug use or other factors for HIV infection. The patient had traveled to Inner Mongolia 1-month prior and had experience weight loss of 6 kilograms during the previous 3 months.

On the day of admission to our hospital, the patient's temperature rose to $38.8^{\circ} \mathrm{C}$ from $37.5^{\circ} \mathrm{C}$ at home, with sweating and arthralgia, but other vital signs were normal. Physical examination revealed a 7x16 $\mathrm{cm}$ non-tender, fluctuant right flank swelling but no visible cutaneous changes. Lumbar flexion movement was limited, hepatosplenomegaly wasn't palpated, and inguinal and axillary lymph nodes were undetectable. Myodynamia of right lower limb weakened from Grade 5 to 3 according to the Frankel grade (13). Pick-up test and right Lasegue sign was positive. Babinski sign was negative on both sides. Nerve damage was Frankel Grade D. Systemic examination was normal.

Laboratory examinations and imaging findings. On admission a specimen of blood was collected and sent for culture. Laboratory examinations demonstrated a white blood cell count of 8,900 cells $/ \mathrm{mm}^{3}$ (normal, 3,500-8,500 cells $/ \mathrm{mm}^{3}$ ) with $65 \%$ neutrophils. Inflammatory markers were elevated significantly, with an erythrocyte sedimentation rate (ESR) of $102 \mathrm{~mm} / \mathrm{h}$ (normal, 0.0-20 mm/h), C-Reactive Protein (CRP) level of $46 \mathrm{mg} / \mathrm{dl}$ (normal, $0.0-10 \mathrm{mg} / \mathrm{dl}$ ) and procalcitonin of $0.43 \mathrm{ng} / \mathrm{ml}$ (normal, 0-0.05 ng/ml). TB-Interferon Gamma Release assay was positive. A Tuberculin Purified Protein Derivative (PPD) skin test was positive, with a $15 \mathrm{~mm}$ diameter (normal, 0.0-5 mm) (14). Hepatic and renal functions were normal. The anti-HIV antibody test was negative. Abdominal ultrasonography didn't indicate any marked changes, with the exception of retroperitoneal multiple liquid dark areas. Chest and hip X-rays were normal, but a lateral radiograph of the lumbar spinal indicated a narrowed disc space and spondylitis at the L4-5 level (Fig. 1A). Computed tomograph (CT) scan indicated vertebral destruction, formation of sequestra and bilateral psoas abscesses and right iliac fossa abscess (Fig. 1B-D). Contrast indicated that the abscesses were clearly peripherally enhanced (Fig. 1E). The magnetic resonance imaging (MRI) of the T2-weighted fat suppression axial demonstrated that the abscess had spread into the spinal canal causing thecal sac compression (Fig. 1F).

Diagnosis and management. According to the aforementioned results, the preliminary diagnosis was lumbar TB combined with psoas abscess. Quadruple anti-tuberculosis drug therapy was initiated empirically [isoniazid $300 \mathrm{mg}$, pyrazinamide $25 \mathrm{mg} / \mathrm{kg}$ (both Shanghai Shangyin Xinyi Pharmaceutical Co., Ltd., Shanghai, China) rifampin $600 \mathrm{mg}$ and ethambutol $20 \mathrm{mg} / \mathrm{kg}$ (both Hutchison Whampoa Guangzhou Baiyunshan Chinese Medicine Co. Ltd., Guangzhou, China) daily]. Due to the intermittent hyperpyrexia and sweating, pyogenic spondylitis was also considered as differential diagnosis, although the patient's white blood cell and neutrophil level was normal. Taken into consideration the patient's travel history to Inner Mongolia, which is one of the major pastoral areas of China (15), prior to a fever, a number of laboratory tests were completed to determine a positive diagnosis of brucellosis. Rose-Bengal Plate Test (RBPT) was positive (16) and as a result, a standard serum agglutination test (SAT) was performed as previously described (17), using the SAT antigen (cat. no. SY0070; Kemin Bio-Technology Ltd., Shanghai, China) which revealed a titer of antibodies to Brucella of 1/80. Following laboratory confirmation of BS, antibiotic treatment was initiated with doxycycline $100 \mathrm{mg}$ twice daily. The blood samples obtained on admission yielded B. melitensis, and a diagnosis of BS was confirmed. During this period the patient's fever and sweating gradually disappeared and lower back pain improved. In order to determine further treatment, a CT-guided percutaneous drainage of the abscess was performed and four drainage catheters were inserted. The pus was sent for culture. The abscesses were aspirated and irrigated with saline solution $(5-10 \mathrm{ml})$ and isoniazid $(200 \mathrm{mg}$ ) twice a day. The culture of the pus was positive for $M$. tuberculosis. Therefore, the definitive diagnosis was spinal TB combined with BS. Drainage catheters were removed when abscesses were minimal $(<30 \mathrm{ml})$, as indicated by a follow-up CT. Single posterior approach surgery, including decompression, debridement, posterior instrumentation and fusion, was subsequently performed under general anesthesia. Inititaly, a CT-guided percutaneous drainage of the abscess was performed and when the abscess was minimal $(<30 \mathrm{ml})$ the drainage catheters were removed. The posterior approach surgery was subsequently performed to remove the abscess located in the intervertebral space and intraspinal canal. During surgery, internal fixation (M8; Medtronic, Minneapolis, MN, USA) was used following decompression and debridement to maintain the stability of the spine. Any residual abscess was treated by postoperative anti-tuberculosis drug therapy. On patient admission anti-tuberculosis drug therapy was initiated for 7 days and the CT-guided percutaneous drainage was performed for 10 days. When the catheters were removed the patient continued quadruple antituberculosis and doxycycline treatment for a further 7 days, then the operation was performed. There was a total of 24 days from hospital admission to surgery. Histopathological examination of the lumbar lesion post surgery revealed granulomatous inflammation and caseous necrosis (Fig. 2), which is consistent with the diagnosis of spinal TB. Tissue sections were fixed in $4 \%$ paraformaldehyde at $4^{\circ} \mathrm{C}$ for $4 \mathrm{~h}$, placed in processing cassettes, dehydrated through a serial alcohol gradient and embedded in paraffin wax blocks. Prior to immunostaining, 5 - $\mu \mathrm{m}$-thick tissue sections were dewaxed in xylene, rehydrated through decreasing concentrations of ethanol and washed in PBS. The sections were stained with hematoxylin and eosin at $20^{\circ} \mathrm{C}$ (hematoxylin for $10 \mathrm{~min}$ and eosin for $30 \mathrm{sec}$ ). Following staining the sections were dehydrated through increasing concentrations of ethanol and xylene. A light microscope was used to examine the tissue sections at magnification, $\mathrm{x} 200$. The patient was at rest for 2 weeks and then began to complete daily activities with a lumbar brace. The patient no longer experienced fever, sweating or arthralgia. Numbness and weakness of the right lower limb disappeared and myodynamia recovered to Grade 5. Treatment with oral doxycycline was completed and the patient was discharged to continued quadruple antituberculosis treatment for 6 months (isoniazid $300 \mathrm{mg}$, rifampin $600 \mathrm{mg}$, ethambutol $20 \mathrm{mg} / \mathrm{kg}$ and pyrazinamide $25 \mathrm{mg} / \mathrm{kg}$ ) and double antituberculosis treatment for a further 6 months (isoniazid $300 \mathrm{mg}$ and rifampin $600 \mathrm{mg}$ ). A follow-up radiograph after 3 months indicated that the internal fixation was in a good position and preliminary vertebral 


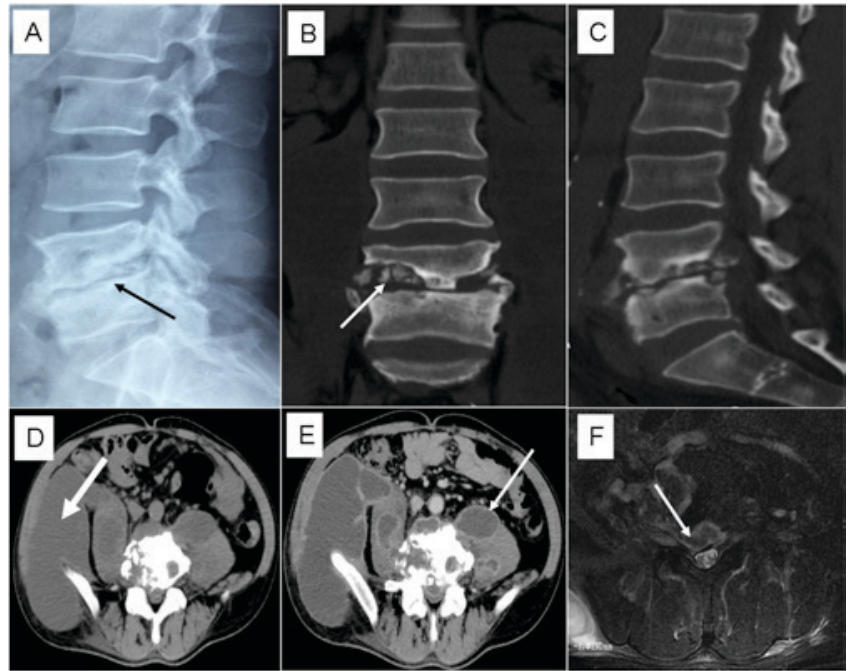

Figure 1. (A) Lateral radiograph of the lumbar spine revealed a narrowed disc space and spondylitis at the L4-5 level (black arrow). (B-D) Computed tomograph scans demonstrated vertebral destruction, formation of sequestra (white thin arrow), bilateral psoas and right iliac fossa abscesses (white thick arrow). (E) Contrast indicated that the abscesses were clearly peripherally enhanced (white arrow). (F) The magnetic resonance imaging of the T2-weighted fat suppression axial revealed that the abscess had spread into the spinal canal causing thecal sac compression (white arrow).

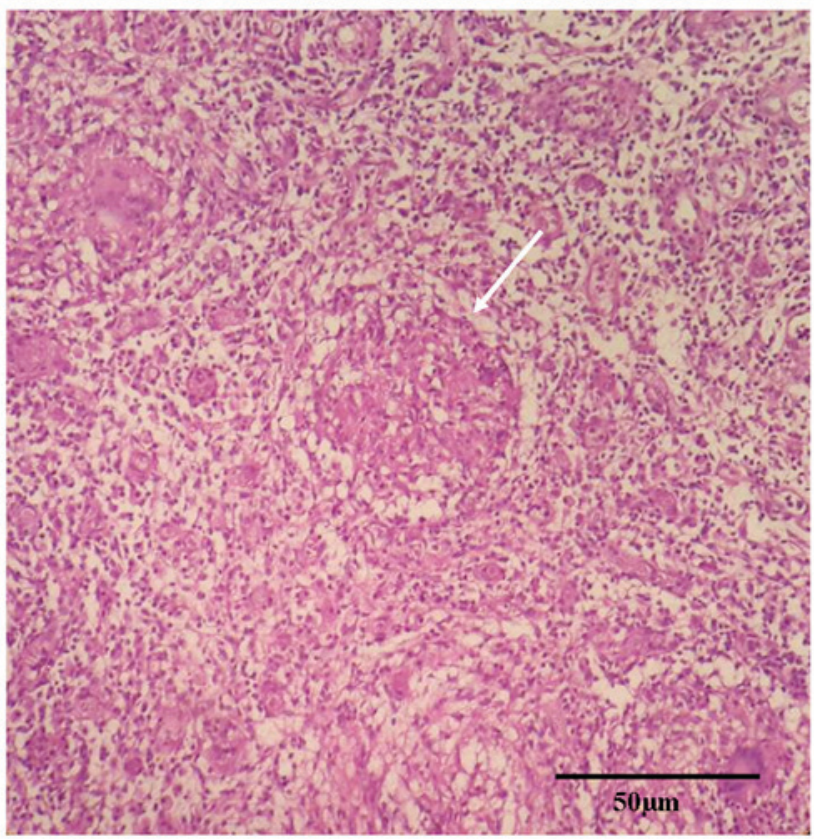

Figure 2. Histopathological slide indicating granulomatous inflammation and caseous necrosis (white arrow). Hematoxylin and eosin staining; magnification $\mathrm{x} 200$.

fusion at L4/5 level, indicating the formation of new bone at the $\mathrm{L} 4 / 5$ disk space.

\section{Discussion}

TB and BS are infectious diseases endemic in certain areas of the world, generally in developing countries $(2,7,18)$ and are relatively common causes of vertebral osteomyelitis (19). Due to similarities in clinical presentation and basic laboratory parameters, a proportion of patients may be misdiagnosed with either TB or BS (18). Despite extensive efforts to control TB, it remains an urgent global health problem, particularly due to the spread of HIV and emergence of multi-drug resistant TB. Human BS is considered to be the most common zoonosis globally, with an annual occurrence of $>500,000$ cases (20). Humans are infected primarily through contact with infected animals or by consuming unpasteurized and unboiled milk or fresh cheese $(10,21)$. BS is listed as a Class II reportable infectious disease by the Centers for Disease Control and Prevention (CDC) of China (Beijing, China). A study by Zhong et al (22) indicated that a total of 155,979 cases were reported in China between 2005 and 2010. BS epidemics are primarily located in the northern provinces of China and inner Mongolia accounted for $50 \%$ of all the reported cases. Fever, night sweating, weight loss, fatigue and elevated ESR and CRP are common clinical features in both infections (22). It is difficult to distinguish TB and BS based on these nonspecific symptoms and laboratory findings; thus, misdiagnosis of BS as TB often occurs in brucellosis low-incidence areas. BS patients may also recover following the use of standard anti-tuberculosis drugs therapy, as antibiotic combination is typical in TB and BS drugs (19,23), and rifampin and streptomycin have notable curative effects in BS and TB. However, etiological treatment based on definitive diagnosis should still be the basic principal. There are a number of subtle differences between the two diseases. Compared with the low-grade fever in TB, intermittent hyperpyrexia is more often observed in BS. Sweating is also more common in BS and the sweat is viscous (18). High values of ESR and CRP often indicate TB and are relative indicators, more useful in evaluating response to treatment (24). Kurtaran et al (19) compared the clinical features of 87 cases with spondylitis of TB and BS, concluding that vertebral destruction and compression, kyphosis, para-spinal abscesses and cord compression were more frequent in spinal TB. This is in agreement with results from previous reports $(23,25)$. Hepatosplenomegaly and lymphadenopathy were more common in BS due to bacteriological replication within the reticuloendothelial system (19). At present, culture of the organism from blood and/or pus remains the gold standard in BS and TB diagnosis and treatment; however, this may take 2-4 weeks and often reveals a false negative result $(10,26)$. RBPT and serum agglutination assessments are available and helpful for preliminary identification. Enhanced awareness for BS is critical to avoid misdiagnosis and delayed diagnosis for clinicians. The co-infection of M. tuberculosis and B. melitensis is rare and the exact mechanism of this co-infection remains unclear. Macrophage dysfunction in TB patients may be associated with this mechanism, as it may promote opportunistic local infections including aspergillosis and other bacterial abscesses. Human protection against $B$. melitensis depends on cell-mediated immunity. However, B. melitensis is able to survive and replicate in macrophages and dendritic cells, evading and destroying innate immunity (27). In theory, it is easier for patients with TB to catch BS, compared with healthy individuals as TB may cause macrophage dysfunction, anemia and hypoproteinemia, which makes individuals more vulnerable to infection.

The standard treatment for BS is doxycycline $100 \mathrm{mg}$ orally twice daily and rifampcine $450 \mathrm{mg} /$ day orally for 
6 weeks $(28,29)$. However other studies indicate that the preferred duration of therapy for BS should be $\geq 3$ months due to high relapse rates (19). There remains no consensus for the duration of anti-tuberculosis drug therapy: Some clinicians support the use of short-term triple drugs for 9 months, while others advocate four-drug therapy for 12 months $(7,30)$. It has been suggested that the duration of therapy should vary according to clinical response and laboratory examinations. Regarding the case presented in the current study, 6 weeks of doxycycline combined with quadruple anti-tuberculosis drug therapy for 12 months was prescribed. Due to the huge abscesses and neurological deficits, a CT-guided percutaneous drainage of the abscess and posterior instrumentation was performed in sequence. This is very different from treatments performed previously (30-33). The CT-guided percutaneous drainage not only removed the abscess and improved the patient's general condition with local chemotherapy (isoniazid), but also avoided the use of anterior or anterolateral approach open surgery, which may lead to postoperative complications. In addition, drainage of pus sent to culture and other laboratory examinations was helpful to reach a definitive diagnosis.

In conclusion, spinal tuberculosis combined with BS is a very rare condition. Overlapping clinical presentation and laboratory parameters of TB and BS may lead to misdiagnosis or delayed diagnosis, specifically in cases of co-infection with TB and BS. Clinicians should strengthen their awareness of this condition for similar patients. Furthermore, the CT-guided percutaneous drainage is effective in the diagnosis and management of spinal TB with abscess. However, it should be noted that as it is possible to relapse with TB and BS it is necessary for the patient to receive regular laboratory and radiological examinations.

\section{Acknowledgements}

The authors of the present study express thanks and appreciation to Xueling Wang for technical assistance in the preparation of the study.

\section{References}

1. Metha JS and Bhojraj SY: Tuberculosis of the thoracic spine. J Bone Joint Surg Br 85: 150, 2003.

2. World Health Organization: Global tuberculosis report 2012. WHO, Geneva, 2012.

3. Samohyl M, Solovic I, Rams R, Hirosova K, Vondrova D, Krajcova D, Nadazdyova A, Filova A and Jurkovicova J: Effect of spa treatment and the epidemiology of tuberculosis in the Slovak republic in the year 2014. Clin Soc Work Health Interven 7 24-35, 2016.

4. Cui X, Ma YZ, Chen X, Cai XJ, Li HW and Bai YB: Outcomes of different surgical procedures in the treatment of spinal tuberculosis in adults. Med Princ Pract 22: 346-350, 2013.

5. Saraf SK and Tuli SM: Tuberculosis of hip: A current concept review. Indian J Orthop 49: 1-9, 2015.

6. Fuentes Ferrer M, Gutiérrez Torres L, Ayala Ramírez O, Rumayor Zarzuelo M and del Prado González N: Tuberculosis of the spine. A systematic review of case series. Int Orthop 36: 221-231, 2012.

7. Moon MS: Tuberculosis of spine: Current views in diagnosis and management. Asian Spine J 8: 97-111, 2014.

8. Polley P and Dunn R: Noncontiguous spinal tuberculosis: Incidence and management. Eur Spine J 18: 1096-1101, 2009.

9. Pawlowski A, Jansson M, Sköld M, Rottenberg ME and Källenius G: Tuberculosis and HIV co-infection. PLoS Pathog 8: e1002464, 2012.
10. Franco MP, Mulder M, Gilman RH and Smits HL: Human brucellosis. Lancet Infect Dis 7: 775-786, 2007.

11. Turgut M, Haddad FS and De Divitiis O: Neurobrucellosis: Clinical, diagnostic and therapeutic features. BMJ 333: 15, 2015.

12. Hadush A and Pal M: Brucellosis-An infectious re-emerging bacterial zoonosis of global importance. Int J Livest Res 3: 28-34, 2013.

13. Frankel HL, Hancock DO, Hyslop G, Melzak J, Michaelis LS Ungar GH, Vernon JD and Walsh JJ: The value of postural reduction in the initial management of closed injuries of the spine with paraplegia and tetraplegia. I. Paraplegia 7: 179-192, 1969.

14. Seibert FB and Dufour EH: Comparison between the international standard tuberculins, PPD-S and old tuberculin. Am Rev Tuberc 69: 585-594, 1954.

15. Bai Y, Han X, Wu J, Chen Z and Li L: Ecosystem stability and compensatory effects in the inner mongolia grassland. Nature 431: 181-184, 2004.

16. Rose JE and Roepke MH: An acidified antigen for detection of nonspecific reactions in the plate-agglutination test for bovine brucellosis. Am J Vet Res 18: 550-555, 1957.

17. Alton GG, Maw J, Rogerson BA and Mcpherson GG: The serological diagnosis of bovine brucellosis: An evaluation of the complement fixation, serum agglutination and rose bengal tests. Aust Vet J 51: 57-63, 1975.

18. Dasari S, Naha K and Prabhu M: Brucellosis and tuberculosis: Clinical overlap and pitfalls. Asian Pac J Trop Med 6: 823-825, 2013.

19. Kurtaran B, Sarpel T, Tasova Y, Candevir A, Saltoglu N, Inal AS and Aksu HSZ: Brucellar and tuberculous spondylitis in 87 adult patients: A descriptive and comparative case series. Infect Dis Clin Prac 16: 166-173, 2008.

20. Dal T, Celen MK, Ayaz C, Dal MS, Kalkanli S, Mert D, Yildirim N, Aktas E and Arserim N: Brucellosis is a major problem: A five years experience. Acta Med Mediterr 29: 665, 2013.

21. Pappas G, Akritidis N, Bosilkovski M and Tsianos E: Brucellosis. N Engl J Med 352: 2325-2336, 2005.

22. Zhong Z, Yu S, Wang X, Dong S, Xu J, Wang Y, Chen Z, Ren Z and Peng G: Human brucellosis in the People's Republic of China during 2005-2010. Int J Infect Dis 17: e289-e292, 2013.

23. Yilmaz E, Parlak M, Akalin H, Heper Y, Ozakin C, Mistik R, Oral B, Helvaci S and Töre O: Brucellar spondylitis: Review of 25 cases. J Clin Rheumatol Pract Rep Dis 10: 300-307, 2004.

24. Ansari S, Ashraf AN, Najib A, Moutaery and Al K: Spinal infections: A review. Neuro Quart 11: 112-123, 2001.

25. Alothman A, Memish ZA, Awada A, Al-Mahmood S, Al-Sadoon S, Rahman MM and Khan MY: Tuberculous spondylitis: Analysis of 69 cases from Saudi Arabia. Spine (Phila Pa 1976) 26: E565-E570, 2001

26. Schaaf HS, Shean K and Donald PR: Culture confirmed multidrug resistant tuberculosis: Diagnostic delay, clinical features, and outcome. Arch Dis Child 88: 1106-1111, 2003.

27. Billard E, Cazevieille C, Dornand J and Gross A: High susceptibility of human dendritic cells to invasion by the intracellular pathogens Brucella suis, B. Abortus, and B. Melitensis. Infect Immun 73: 8418-8424, 2005.

28. Hartady T, Saad MZ, Bejo SK and Salisi MS: Clinical human brucellosis in Malaysia: A case report. Asian Pac J Trop Dis 4: 150-153, 2014.

29. Human brucellosis: Systematic review and meta-analysis of randomised controlled trials. BMJ 336: 701, 2008.

30. Sundararaj GD, Behera S, Ravi V, Venkatesh K, Cherian VM and Lee V: Role of posterior stabilisation in the management of tuberculosis of the dorsal and lumbar spine. J Bone Joint Surg Br 85: 100-106, 2003.

31. Chen WJ, Wu CC, Jung CH, Chen LH, Niu CC and Lai PL: Combined anterior and posterior surgeries in the treatment of spinal tuberculous spondylitis. Clin Orthop Relat Res: 50-59, 2002.

32. Liu P, Sun M, Li S, Wang Z and Ding G: A retrospective controlled study of three different operative approaches for the treatment of thoracic and lumbar spinal tuberculosis: Three years of follow-up. Clin Neurol Neurosurg 128: 25-34, 2015.

33. Li J, Li XL, Zhou XG, Zhou J and Dong J: Surgical treatment for spinal tuberculosis with bilateral paraspinal abscess or bilateral psoas abscess: One-stage surgery. J Spinal Disord Tech 27: E309-E314, 2014. 